\title{
Morbimortalidade pela Covid-19 segundo raça/cor/etnia: a experiência do Brasil e dos Estados Unidos
}

\author{
Covid-19 morbimortality by race/skin color/ethnicity: the Brazilian \\ and the North American experiences
}

\author{
Edna Maria de Araújo', Kia Lilly Caldwel|2 ${ }^{\mathbf{2}}$, Márcia Pereira Alves dos Santos ${ }^{\mathbf{3}}$, Ionara Magalhães \\ de Souza ${ }^{\mathbf{4}}$, Patrícia Lima Ferreira Santa Rosa ${ }^{\mathbf{5}}$, Andreia Beatriz Silva dos Santos ${ }^{\mathbf{1}}$ Luís Eduardo \\ Batista 6
}

DOI: 10.1590/0103-11042020E412

1 Universidade Estadual de Feira de Santana (Uefs) - Feira de Santana (BA), Brasil.

ednakam@gmail.com

2 University of North Carolina (UNC),

Department of African, African American, and Diaspora Studies - Chapel Hill (Carolina do Norte),

Estados Unidos da

América.

3 Universidade Federal do Rio de Janeiro (UFRJ) - Rio de Janeiro (RJ), Brasil.

4 Universidade Federal do Recôncavo da Bahia (UFRB) - Santo Antônio de Jesus (BA), Brasil.

${ }^{5}$ Fundação Instituto de Educação de Barueri (Fieb) - Barueri (SP), Brasil.

6 Secretaria de Estado da Saúde de São Paulo (SES-SP), Coordenadoria de Controle de Doenças (CCD) - São Paulo (SP), Brasil.
RESUMO Este estudo objetivou descrever a experiência do Brasil e dos Estados Unidos da América (EUA) em relação aos dados de morbimortalidade por Covid-19, segundo a raça/cor/etnia. Para isso, procurou-se descrever os fatores envolvidos no tratamento e divulgação dos dados de morbimortalidade por essa patologia nos dois países. Foram analisados boletins epidemiológicos divulgados pelo Ministério da Saúde, resultados parciais da Pesquisa Nacional por Amostra de Domicílio (PNAD) Covid-19 realizada pelo Instituto Brasileiro de Geografia e Estatística (IBGE) no Brasil e o estado da arte em saúde sobre os impactos da pandemia nos EUA, sob a perspectiva de raça/cor/etnia. Apesar da baixa qualidade da informação em saúde referente à morbimortalidade da população negra por Covid-19, os resultados desvelam iniquidades raciais em saúde para essa doença, ratificando o racismo estrutural/institucional em ambos os países. Como contribuição, enfatiza-se a necessidade de qualificar os dados sobre raça/ cor/etnia, relacionando-os com idade, local de moradia, tipo de residência, acesso a saneamento básico, ocupação, entre outros determinantes sociais que, sabidamente, impactam no modo de adoecer e morrer pela Covid-19, a fim de viabilizar estratégias e políticas públicas verdadeiramente promotoras da equidade.

PALAVRAS-CHAVE Raça e saúde. Etnia e saúde. Morbimortalidade. Sistema de informação em saúde. Racismo.

\begin{abstract}
This study sought to describe the experience of Brazil and the United States of América (USA) in relation to Covid-19 morbimortality data according to race/skin color/ethnicity. Toward this end, it seeks to describe the factors involved in the treatment and dissemination of the morbimortality data for such pathology in the two countries. The analysis includes epidemiological bulletins released by the Brazilian Ministry of Health, partial results from Brazil's National Household Sample Survey (PNAD) for Covid-19 collected by the Brazilian Institute of Geography and Statistics (IBGE), and state-of-the-art health data about the impact of the pandemic in the USA, from the perspective of race/skin color/ethnicity. Despite the low quality of health information on Covid-19 morbimortality of black population, the results corroborate racial inequities in health for the disease, confirming the existence of structural and institutional racism in both countries. This article highlights the need to qualify data about race/skin color/ethnicity, by relating them to age, place of residence, type of residence, access to basic sanitation, and occupation, among other social determinants that impact how individuals become ill and die from Covid-19, in order to enact strategies and public policies that truly promote equity.
\end{abstract}

KEYWORDS Race and health. Ethincity and health. Morbidity and mortality. Health informaton system. Racism. 


\section{Introdução}

Em 11 de março de 2020, a Organização Mundial da Saúde decretou emergência em saúde pública de interesse internacional por coronavírus ${ }^{1}$, agente causal da Covid19. Esta doença apresenta um espectro diversificado de sintomas, variando de leves a graves. A Síndrome Respiratória Aguda Grave (SRAG) pode ser causada por diversos agentes etiológicos, entre eles, o novo coronavírus (SARS-CoV-2). A SRAG possui letalidade variada a depender dos fatores condicionantes, como a existência de comorbidades, a idade, o modo de vida e o acesso aos serviços de saúde ${ }^{2}$.

Neste contexto de emergência sanitária global, os modos de viver estão marcadamente transversalizados pela falta de estabilidade socioeconômica traduzida pela perda de salários, hipossuficiência financeira, falta de recursos, acrescida da falta de moradia e de infraestrutura que garanta as condições de sobrevivência em meio à crise econômica, aliada ainda à dificuldade de acesso a serviços de saúde ${ }^{3}$. Esses fatores explicam, em parte, as maiores taxas de morbimortalidade das populações em situação de vulnerabilidade. Em países marcados por profundas desigualdades sociais, os impactos da pandemia da Covid-19 têm sido ainda mais devastadores. Nessa direção, sabe-se que as populações negras sobreacumulam os efeitos mais deletérios.

Para a população negra, a pandemia da Covid-19 atualiza as insolvências do passado histórico a uma contemporaneidade conjuntural, cujo cerne é o racismo em suas diferentes dimensões ${ }^{4,5}$. Soma-se a isso, a precariedade do registro da raça/cor/etnia nos sistemas de informação em saúde e nos relatórios de informação em saúde ${ }^{6,7}$. Essa falta de informação por raça/cor/etnia representa não só um grande problema à implementação de políticas públicas de saúde, como também caracteriza a baixa adesão à Política Nacional de Saúde Integral da População Negra no território brasileiro ${ }^{8}$.
O racismo foi constituído historicamente, com base na crença da inferioridade de um grupo em relação ao outro, enquanto prática de exclusão do diferente e exercício do poder ${ }^{9}$ como um sistema estruturado e estruturante. Ele é um determinante social de saúde que afeta adversamente a saúde das populações e representa a causa fundamental das iniquidades de acesso aos bens, recursos e oportunidades em uma realidade global ${ }^{10}$.

A necessidade de produção e análise de dados desagregados na agenda de todos os países para superar o racismo representou importante pauta da Conferência Mundial contra o Racismo, Xenofobia e Intolerâncias Correlatas realizada em Durban, em 2001"11, da qual o Brasil é signatário.

O País incluiu o quesito raça/cor nos sistemas de informação em saúde somente a partir de $1990^{12}$, mas alguns de seus sistemas nacionais de informação em saúde no Brasil refutam as normativas que recomendam a obrigatoriedade da presença e do preenchimento do quesito raça/cor nos instrumentos do Sistema Único de Saúde (SUS) ${ }^{\mathbf{1 3}, 14}$. Por outro lado, ainda que em alguns instrumentos conste o campo raça/cor, a incompletude desse campo, a alta frequência do preenchimento da categoria 'Ignorado' ou ainda a ausência de análises com estratificação racial caracterizam a desimportância desse dado como proxy social e expressam o racismo institucional. A não inclusão e a incompletude do campo raça/cor configuram grande barreira ao enfrentamento do racismo e à promoção da equidade em saúde no País ${ }^{15}$.

Os Estados Unidos da América (EUA) coletam dados desagregados por raça/etnia há décadas ${ }^{16}$. As informações sobre a vigilância da saúde pública e demais informações do País, bem como os dados sobre raça/ cor/etnia, são obtidos nos departamentos estaduais e no National Center for Health Statistics (NCHS) do Centers for Disease Control and Prevention (CDC), responsável pelas estatísticas sobre a saúde da população. O Relatório do workshop que ocorreu em Atlanta em 1993, intitulado 'The Use 
of Race and Ethnicity in Public Health Surveillance', que discutiu o papel da raça e etnia na vigilância da saúde pública, identificou os esforços da vigilância para incluir todas as pessoas, a falta de consenso para definição da raça e etnia e também a limitação dos sistemas de vigilância em saúde pública em apresentar, entre os indicadores publicizados, os dados relativos à raça/cor/etnia ${ }^{17}$.

Em ambos os contextos, a construção de indicadores por raça/cor/etnia tem impactos significativos na condução das ações em saúde e redução das iniquidades. Nesse sentido, desagregar dados que informam classe, gênero, raça/cor/ etnia e lugar de residência é fundamental para melhor evidenciar as iniquidades em saúde ${ }^{\mathbf{1 8}}$.

A insuficiência e a não desagregação de dados sobre morbimortalidade por Covid-19, segundo raça/cor/etnia, portanto, revela-se como grande preocupação nos contextos brasileiro e estadunidense. Dessa forma, este estudo objetivou descrever a experiência do Brasil e dos EUA em relação aos dados de morbimortalidade por Covid-19 segundo a raça/cor/etnia.

\section{Material e métodos}

Trata-se de estudo descritivo, exploratório que buscou descrever os aspectos envolvidos no tratamento e na divulgação dos dados de morbimortalidade por Covid-19 no Brasil e nos EUA. Foram analisados boletins epidemiológicos divulgados pelo Ministério da Saúde, resultados parciais da Pesquisa Nacional por Amostra de Domicílio (PNAD) Covid-19 realizada pelo Instituto Brasileiro de Geografia e Estatística (IBGE) no Brasil e o estado da arte em saúde sobre os impactos da pandemia nos EUA, sob a perspectiva de raça/cor/etnia.

Foram analisados 21 boletins epidemiológicos divulgados pelo Ministério da Saúde nos quais foram investigados aspectos da produção e divulgação dos dados desagregados por raça/cor ao longo do período de janeiro a julho de 2020. Os boletins foram obtidos nos seguintes links: https://coronavirus.saude. gov.br/boletins-epidemiologicos e https:// coronavirus.saude.gov.br/2 2 .

A PNAD-Covid-1919 teve como objetivo estimar o número de pessoas com sintomas referidos associados à síndrome gripal e monitorar os impactos da pandemia da Covid-19 no mercado de trabalho brasileiro. A coleta da pesquisa teve início em 4 de maio de 2020, em que se entrevistou por telefone aproximadamente 48 mil domicílios por semana, totalizando cerca de 193 mil domicílios por mês. O questionário está dividido em saúde e trabalho. Nas questões de saúde, foram entrevistados todos os moradores que apresentaram sintomas da Covid-19.

\section{Caracterização demográfica e social do Brasil e dos EUA}

\section{BRASIL}

O Brasil ocupa a quinta maior população no mundo, estimada em 211.293 milhões de habitantes, classificada em brancos (45,22\%), pardos $(45,06 \%)$, pretos $(8,86 \%)$, amarelos $(0,47 \%)$ e indígenas $(0,38 \%)^{20}$. A população negra brasileira é constituída por pretos e pardos (53,92\%). Essa junção se justifica, estatisticamente, pelo fato de esses dois grupos apresentarem aspectos socioeconômicos similares e, teoricamente, por se constituírem alvo de discriminação ${ }^{21}$.

$\mathrm{O}$ Brasil corresponde à maior economia da América Latina e à sétima economia do mundo. Apresenta renda per capita de $\mathrm{R} \$$ 1.439,00 (2019), todavia 7,4\% dos brasileiros vivem em extrema pobreza. O País ocupa a $10^{a}$ posição de um conjunto de 143 países no ranking da desigualdade. Em termos de concentração de renda, o Brasil representa o segundo país do mundo com a maior desigualdade na distribuição de renda ${ }^{\mathbf{2 2}}$.

Em relação à assistência à saúde no Brasil, em 1988, foi instituído o SUS com a promulgação da Constituição Federal Brasileira. O 
SUS tem a missão de prover saúde para todos os brasileiros, e a sua expressão máxima é saúde como direito de todos e dever do Estado ${ }^{23}$. Além desse sistema público nacional (e universal), há no Brasil a assistência médica supletiva, que cobre cerca de $25 \%$ da população, ou seja, a grande maioria dos brasileiros depende exclusivamente do SUS.

\section{ESTADOS UNIDOS}

Em 2019, a estimativa populacional dos EUA correspondeu a 328.239 .523 habitantes, configurando-o como o terceiro país mais populoso do planeta. A composição racial e a origem hispânica distribuem-se em brancos (76,3\%), afro-americanos (13,4\%), índios americanos e nativos do Alasca (1,3\%), asiáticos $(5,9 \%)$, havaianos nativos e outras ilhas do Pacífico $(0,2 \%)^{\mathbf{2 4}}$.

Os EUA não têm um sistema de saúde universal. Possuem o Medicare e Medicaid, que são programas de saúde do governo federal que foram criados em 1965, para pessoas vivendo em situação de pobreza. Em 23 de março de 2010, o então presidente Barack Obama sancionou a Lei de expansão desses programas, denominada Affordable Care Act (ACA) ou 'Obamacare', cujo objetivo é prestar assistência à significativa parte da população do país que não tem seguro de saúde, mas que até agora não foi aprovada em 14 estados por motivos políticos ${ }^{25,26}$. Em 10 desses estados, a população negra constitui mais de $15 \%$ da população total27. $\mathrm{Na}$ administração de Donald Trump, tem havido várias mudanças no ACA, assim como tentativas de enfraquecê-lo, mesmo durante a pandemia da Covid-1928.

A situação econômica nos EUA, antes e durante a pandemia, tem tido um maior impacto negativo para a população negra, incluindo o nível de desemprego que é duas vezes maior em comparação à população branca. Durante a pandemia, a proporção de perda de emprego entre negros é de $44 \%$; e entre brancos é de $38 \%{ }^{29}$.

\section{Resultados}

\section{A experiência do registro raça/cor/ etnia no Brasil durante a pandemia da Covid-19}

\section{BOLETINS EPIDEMIOLÓGICOS (BE) PUBLICADOS PELO MINISTÉRIO DA SAÚDE DO BRASIL}

Os dados desagregados por raça/cor passaram a ser divulgados a partir do Boletim Epidemiológico 09 (BE-09) respectivo à semana epidemiológica $\mathrm{n}^{0} 15$, entre 04 e 10 de abril, e que se referiu às hospitalizações por SRAG. Na sequência, o BE-10 divulgou dados de óbitos por Covid-19 e dados de hospitalizações por SRAG segundo raça/cor. Os BE-11, 12, 13, 14 e 15 apresentaram dados sobre SRAG, desagregados por raça/cor. Porém, somente a partir do BE-13 os dados sobre hospitalizações por SRAG foram apresentados como decorrência da Covid-19 por raça/cor.

Os BE-16 e 17 são destacados como Boletins Epidemiológicos Especiais (BEE), referem-se à mesma semana epidemiológica, no 21 (17 a 23 de maio de 2020) e apresentam casos confirmados para Covid-19, desagregados por raça/cor, separados por óbitos e hospitalizações. Adicionalmente, o BEE-16 detalhou ações relativas à vigilância laboratorial para enfrentamento da Covid-19, e o BEE-17 apresentou dados de gestantes, crianças e adolescentes confirmados para Covid-19 desagregados por raça/cor, tendo como fonte o Sistema de Informações de Vigilância Epidemiológica da Gripe, o Sivep-Gripe. No BEE-16, os dados foram divulgados segundo porcentagens e números absolutos. Os dados de hospitalizações de SRAG por Covid-19 aparecem somente em gráficos com porcentagens e com uma nota de rodapé explicando que 14.698 casos foram excluídos da análise devido ao campo raça/cor ter sido registrado como ignorado. Esse padrão se repete nos demais boletins. Os dados de óbitos também são apresentados em gráfico, 
nos quais é possível visualizar que $43,1 \%$ ocorreram entre pessoas de raça/cor branca, seguido da raça/cor parda (47,3\%) e preta (7,5\%). Entre esses óbitos, 4.425 tiveram a raça/cor ignorada e não foram incluídos na análise.

O BEE-17 mostra a distribuição percentual das hospitalizações por SRAG por Covid-19 segundo raça/cor. Assim, 49,0\% das hospitalizações ocorreram entre pessoas de raça/cor branca, seguidas da raça/cor parda $(42,0 \%)$ e preta (7,1\%). Entre esses casos, 19.226 tiveram a variável raça/cor ignorada e não foram incluídas na análise. Já em relação aos óbitos de SRAG por Covid-19, observou-se que 49,6\% dos óbitos ocorreram entre pessoas de raça/cor parda, seguidos da raça/cor branca $(41,0 \%)$ e preta (7,4\%). Entre esses óbitos, 6.245 tiveram a variável raça/cor ignorada e foram excluídos da análise. Adicionalmente, o BEE-17 mostrou que gestantes negras representavam $48,0 \%$ dos casos, enquanto as gestantes brancas, $24,2 \%$. A frequência relativa dos casos em que a raça/cor foi ignorada ou estava em branco correspondeu a $26,6 \%$. Em relação aos óbitos, as gestantes negras representaram $55,5 \%$ dos casos, enquanto as gestantes brancas totalizaram 13,9\%; e 30,6\% corresponderam aos casos cuja raça/cor foi ignorada ou estava em branco. Em relação às crianças e aos adolescentes com Covid-19, as crianças negras representaram $55,3 \%$ dos casos, enquanto $59,5 \%$ eram adolescentes, não havendo referência aos dados ignorados.

Já no BEE-21, observou-se que, entre as gestantes pretas, pardas ou indígenas, a proporção de casos de 'SRAG' foi 49,4\%; e de 'SRAG devido à Covid-19' foi de 52,6\%. No caso dos óbitos entre gestantes por SRAG decorrente da Covid-19, comparando os BEE-17 e 21, observou-se, um aumento expressivo em quase todas as categorias raciais, excetuando-se as indígenas, sendo as gestantes pardas as mais acometidas (gráfico 1). Diabetes e cardiopatias foram as comorbidades mais prevalentes entre as gestantes que evoluíram a óbito por SRAG decorrente da Covid-19.

Gráfico 1. Distribuição de óbitos de gestantes por SRAG por Covid-19, segundo raça/cor entre os BEE 17 e 21, Brasil, 2020

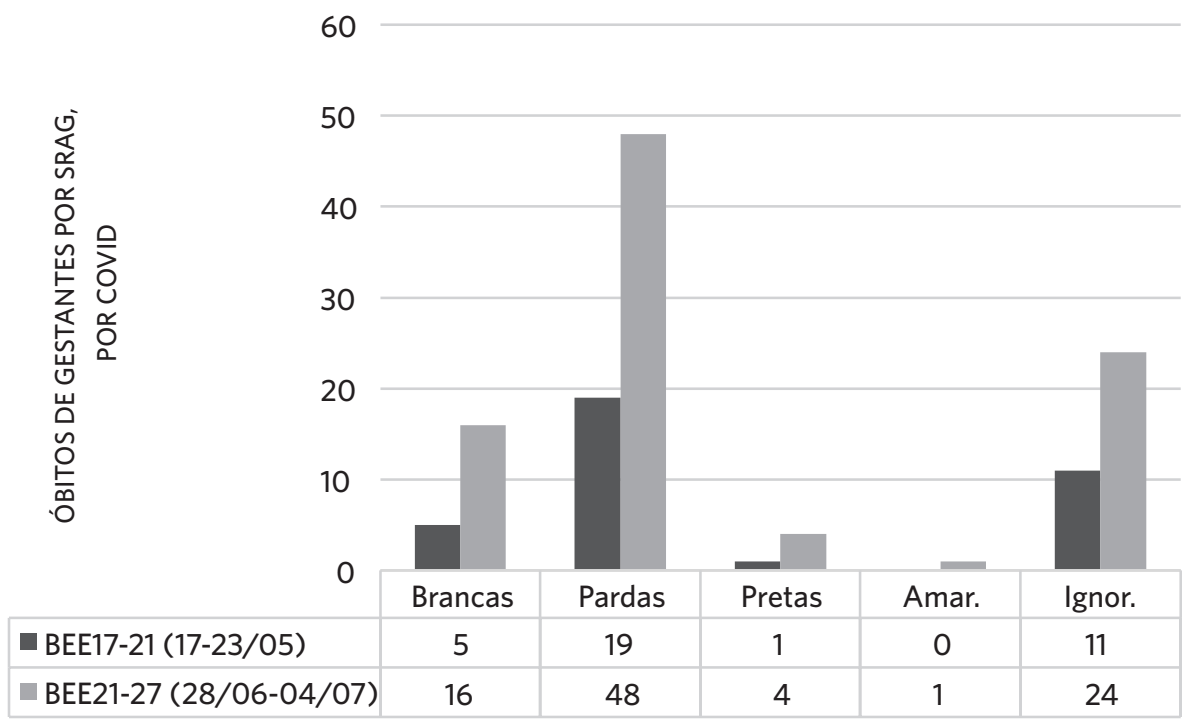

Fonte: Elaboração própria. 
A partir do BEE-18, os dados passaram a ser apresentados em tabelas com valores absolutos, não mais com valores percentuais. Destaca-se, ainda, no BEE-18, a informação de que os dados não contabilizados se referiam aos casos 'sem informação' sobre o campo raça/cor. O BEE-18 contém um eixo denominado 'Resultados do Vigitel Covid-19', em que é descrita a presença de Doenças Crônicas Não Transmissíveis e sua interface com os comportamentos adotados ou não por uma amostra de 2 mil pessoas, sobre as formas de prevenção contra a Covid-19. contudo, não é feita nenhuma referência à variável raça/cor.
Desde o BEE-18 até o BEE-21, correspondentes ao período entre 07 de junho e 04 de julho, foram apresentados dados de hospitalizações e óbitos desagregados por raça/cor somente em números absolutos. A partir destes, foram calculados os percentuais, evidenciando que pessoas negras foram mais hospitalizadas do que pessoas brancas. Por exemplo, no BEE-19, evidenciou-se que pessoas negras $(35,5 \%)$ foram hospitalizadas mais do que pessoas brancas (27,7\%), e os casos ignorados ou sem informação do campo raça/cor corresponderam a $41,9 \%$.

Gráfico 2. Distribuição das hospitalizações por SRAG, segundo raça/cor, entre Boletins Epidemiológicos 09 e 21, Brasil, 2020

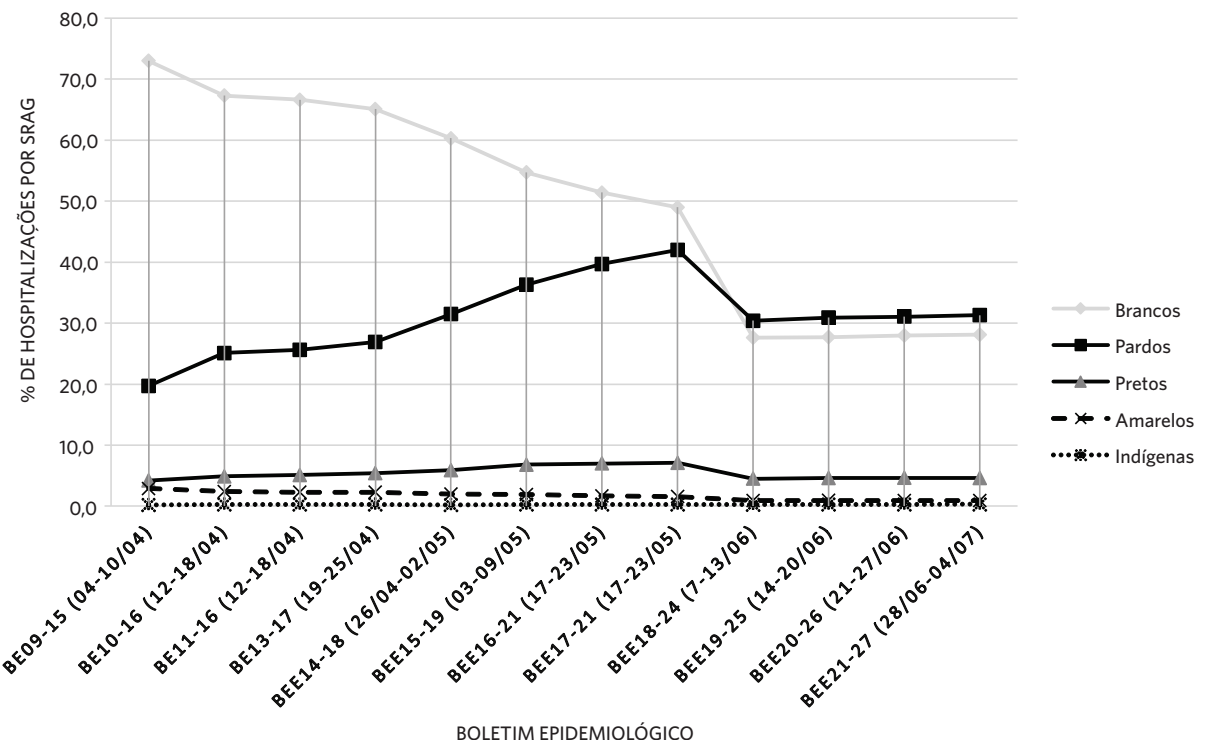

Fonte: Elaboração própria.

Os dados divulgados referentes às hospitalizações por SRAG, desde o BE-09 até o BEE-21, segundo a raça/cor, são mostrados no gráfico 2. Nele, é possível observar que, no início da pandemia no Brasil, a porcentagem de hospitalizações entre pessoas de raça/cor branca foi maior e decresceu ao longo do período; ao passo que a porcentagem de hospitalizações entre pessoas negras mostrou-se crescente ao longo do período. A partir da semana epidemiológica 21 (BEE17), observa-se uma redução expressiva dos percentuais de hospitalização para todos os grupos raciais. 
Os dados relativos aos óbitos por Covid19 publicados desde o BE-10 até o BEE-21, segundo raça/cor, estão expressos no gráfico 3. Observou-se o mesmo padrão referente às hospitalizações, ao passo que a porcentagem de óbitos entre pessoas pardas cresceu no mesmo período, ultrapassando o percentual entre pessoas brancas.

Gráfico 3. Coeficiente de infecção pela Covid-19, segundo raça/cor/etnia, CDC, 13 de junho, Estados Unidos, 2020

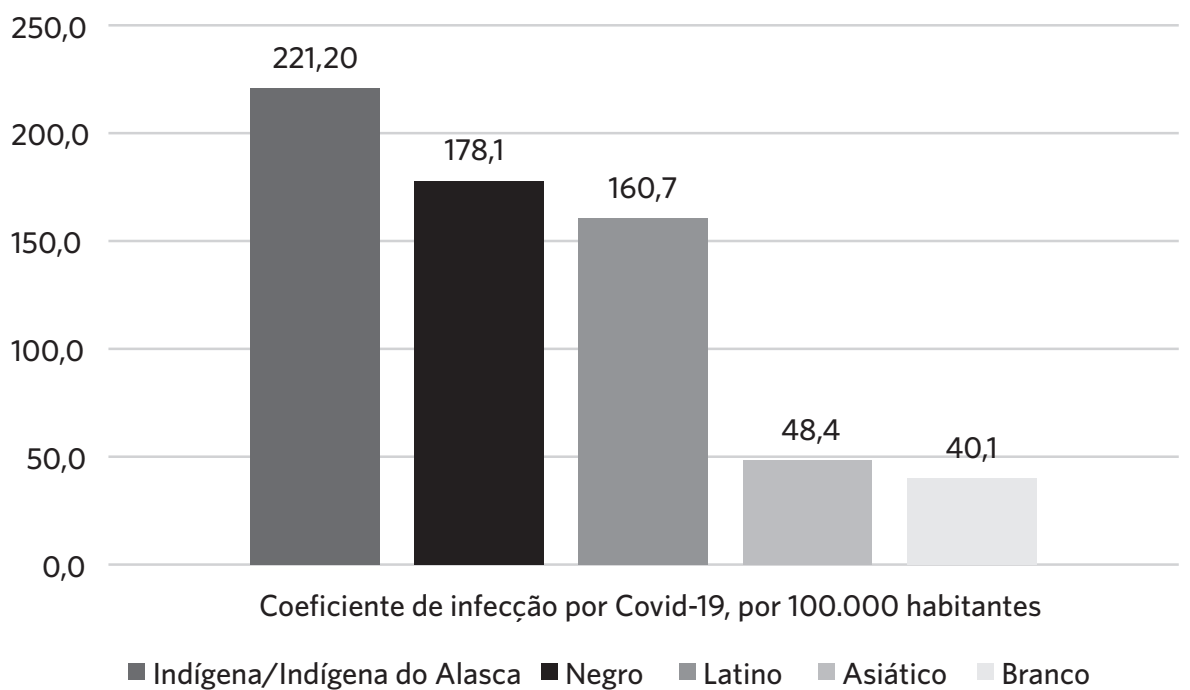

Fonte: Elaboração própria.

\section{A PESQUISA PNAD/COVID-19}

Tabela 1. Resultados parciais da pesquisa IBGE, PNAD - Covid-19, segundo raça/cor

\begin{tabular}{|c|c|c|c|}
\hline \multirow[b]{2}{*}{ Variáveis analisadas } & \multicolumn{3}{|r|}{ Raça/Cor } \\
\hline & Total & Branca & Preta ou parda \\
\hline $\begin{array}{l}\text { Número de pessoas que apresentaram algum sintoma por mil } \\
\text { pessoas }\left(\mathrm{CV}^{*} \%\right)\end{array}$ & $24.012(1,0)$ & $9.675(1,4)$ & $13.981(1,2)$ \\
\hline Distribuição das pessoas que apresentaram algum sintoma (\%) & 100,0 & 40,3 & 58,2 \\
\hline $\begin{array}{l}\text { Percentual de pessoas que apresentaram algum dos sintomas no } \\
\text { total da população (\%) (CV*\%) }\end{array}$ & $11,4(1,0)$ & $10,4(1,4)$ & $12,1(1,2)$ \\
\hline $\begin{array}{l}\text { Pessoas que procuraram atendimento de saúde em hospital por mil } \\
\text { pessoas }\left(C V^{\star} \%\right)\end{array}$ & $1.118(3,6)$ & $416(5,7)$ & $686(4,3)$ \\
\hline $\begin{array}{l}\text { Distribuição das pessoas que procuraram atendimento de saúde } \\
\text { em hospital (\%) }\end{array}$ & 100,0 & 37,2 & 61,4 \\
\hline $\begin{array}{l}\text { Pessoas que foram internadas em hospital e ficaram sedadas, intu- } \\
\text { badas e com respiração artificial por mil pessoas }\left(\mathrm{CV}^{*} \%\right)\end{array}$ & $31(19,7)$ & $14(29,7)$ & $17(22,8)$ \\
\hline $\begin{array}{l}\text { Distribuição das pessoas que foram internadas em hospital e fica- } \\
\text { ram sedadas, intubadas e com respiração artificial (\%) }\end{array}$ & 100,0 & 45,1 & 54,9 \\
\hline
\end{tabular}


Tabela 1. (cont.)

\begin{tabular}{lrrrr}
\hline & & & & Raça/Cor \\
\cline { 3 - 5 } Variáveis analisadas & & Total & Branca & Preta ou parda \\
\hline Plano de Saúde Médico - População residente por mil & Total & $210.869(0,0)$ & $92.967(0,5)$ & $115.475(0,4)$ \\
pessoas (CV*\%) & Sim & $61.541(0,9)$ & $36.504(1,3)$ & $24.227(0,1)$ \\
& Não & $148.761(0,4)$ & $56.196(0,6)$ & $90.977(0,5)$ \\
Plano de Saúde Médico - Distribuição da população resi- & Total & 100 & 100,0 & 100,0 \\
dente (\%) & Sim & 29,3 & 39,4 & 21,0 \\
& Não & 70,7 & 60,6 & 79,0 \\
\hline
\end{tabular}

Fonte: IBGE - PNAD - Covid-19/202019

${ }^{*} \mathrm{CV}$ - Coeficiente de Variação.

Os dados apresentados na tabela 1 mostram as frequências absolutas (n), relativas (\%) e o Coeficiente de Variação (CV) da pesquisa PNAD-Covid-19 segundo raça/cor. Os resultados corroboram maior acometimento da população negra pela Covid-19 e maior utilização do SUS por essa população para o cuidado à saúde, já que os dados relacionados com as pessoas negras mostraram maior proporção para os sintomas da Covid-19 e maior procura por atendimento em unidades de saúde ou hospitais públicos com Unidade de Terapia Intensiva (UTI). Houve predominância da população branca como portadora de planos de assistência médica supletiva.

\section{A EXPERIÊNCIA DE REGISTRO DE RAÇA/COR/ ETNIA NA PANDEMIA NOS EUA: O QUE DIZ O ESTADO DA ARTE?}

Disparidades étnico-raciais com relação ao número de casos e óbitos por Covid-19 ficaram evidentes nos EUA no final de março de 2020.

Em abril de 2020, vários pesquisadores, políticos e pessoas que advogam no setor de saúde pressionaram o governo federal e o CDC para acompanhar e divulgar os dados sobre Covid-19, segundo raça/cor/etnia, assim como por gênero e status socioeconômico. O CDC concordou em divulgar os dados por raça/cor/ etnia em 17 de abril 2020. No dia 22 de abril 2020, o CDC publicou seu primeiro relatório sobre o impacto da Covid-19 segundo raça/ cor/etnia ${ }^{30}$.

Dados de abril a junho de 2020 na cidade de Nova Iorque revelaram que os afro-americanos constituem $22 \%$ da população, mas o percentual de óbitos entre eles correspondeu a $28 \%{ }^{31,32}$. No estado de Nova Iorque, essa população constituiu $9 \%$, mas concentrou $18 \%$ das mortes por Covid-1932.

Em Milwaukee County, Wisconsin, os negros representam $26 \%$ da população, e o percentual de óbitos por Covid-19 entre eles correspondeu a $73 \%{ }^{33}$.

Em Dougherty County, Georgia, os negros são $69 \%$ da população, e $81 \%$ das mortes por Covid-19 ocorreram entre pessoas negras ${ }^{33}$. $\mathrm{Na}$ cidade de St. Louis, os negros representaram $47 \%$ da população, mas, até o começo de maio, concentravam $75 \%$ dos casos de Covid-1934.

Um estudo realizado por Millet et al. ${ }^{33}$ constatou que, em 78\% dos dados dos departamentos estaduais de saúde, faltavam informações desagregadas por raça/cor/etnia. Este estudo também apontou que, no Estado da Georgia, 62,7\% dos dados sobre casos de Covid-19 não incluíram a informação raça/cor/etnia ${ }^{33}$. No estado de Pennsylvania, em $74 \%$ dos casos, também não havia essa informação. Ainda, de um total de 3.141 casos registrados nos relatórios de laboratório Covid-19 do Connecticut State Department of Public Health, mais de 50\% não apresentavam dados sobre raça/cor/etnia ${ }^{3}$. 
Dados do CDC de junho 2020 evidenciaram grandes disparidades nas taxas de internação hospitalar por raça/cor/etnia ${ }^{35}$. O grupo mais afetado tem sido a população indígena norte-americana seguido da população negra. Comparando-se os dados de maio e junho, a taxa de infecção por Covid-19 cresceu de 142 para 178,1/100 mil habitantes ${ }^{30,35}$. Nos EUA, a população indígena é o segmento com a maior taxa de infecção pelo novo coronavírus por 100 mil habitantes (gráfico 3).

Nos EUA, a John Hopkins University elaborou um portal para monitoramento e divulgação de dados e tendências relativos à pandemia. Até o final de junho de 2020, 3 estados não reportaram casos confirmados por raça/cor/etnia, 5 estados não registraram dados de óbitos e apenas 4 (8\%) dos 50 estados estadunidenses reportaram dados sobre testagem com a identificação racial dos indivíduos ${ }^{36}$.

\section{Discussão}

Ao serem analisados os $\mathrm{BE}$, os resultados parciais da pesquisa oficial realizada pelo IBGE no Brasil e o estado da arte em saúde sobre os impactos da Covid-19 nos EUA sob a perspectiva de raça/cor/etnia, observaram-se similaridades nos dois países, a saber:

- o impacto diferenciado da Covid-19 em ambas as realidades mostrou as desigualdades no setor saúde, assim como as desigualdades sociais e econômicas mais amplas. $\mathrm{O}$ impacto maior do vírus na população negra não corroborou a ideia de que a Covid-19 seria uma doença que afeta igualmente todos os grupos sociais;

- vigora uma política explícita de invisibilização de dados que desvelam iniquidades étnico-raciais nesses dois países;

- foi imprescindível a sociedade tensionar para que houvesse a divulgação de dados desagregados por raça/cor/etnia, mesmo em contexto de pandemia;

- quando os governos do Brasil e dos EUA resolveram divulgar os dados por raça/cor/ etnia, esses dados mostram-se de baixa qualidade e inviabilizam análises robustas;

- mesmo com a baixa qualidade dos dados informados, segundo raça/cor/etnia, é possível evidenciar diferenciais no perfil de adoecimento e mortalidade por Covid-19, já que se tem observado que, mesmo quando há predominância de hospitalizações na população branca, registra-se maior incidência de morte na população negra.

No Brasil, a heteroclassificação corresponde ao aspecto determinante das relações sociais, e a evidência científica de que raças não existem não atenua os efeitos do racismo, haja vista que não se recorre à carga genética como base para as relações ${ }^{37}$. Já nos EUA, prevalece a origem genética. Nesse sentido, a lei Jim Crow, instaurada na década de 1930, estabelecia o regime denominado one drop (uma gota de sangue) ou hypo-descent (descendência mínima), segundo o qual a carga genética era determinante no processo de classificação racial. Essas delimitações fundamentam os tipos de preconceitos vigentes no Brasil e nos EUA. No Brasil, as relações raciais são pautadas nos atributos físicos e compõem o preconceito racial de marca, ao passo que, nos EUA, a classificação racial embasada na ascendência constitui o preconceito racial de origem ${ }^{38}$.

No tocante à invisibilização dos dados por raça/cor/etnia no Brasil e nos EUA, é intrigante a similaridade entre esses dois países, já que também no Brasil o percentual de óbitos por Covid-19 entre os negros é superior à composição populacional por região. Enquanto cerca de $54,8 \%$ da população brasileira é composta por negros ${ }^{39}$, entre os que morreram por Covid-19 e tiveram a raça/cor/ etnia identificada como pardos e pretos, esse 
percentual foi de $61 \%$. No Norte, o percentual de óbitos por essa doença entre negros foi de $86 \%$, sendo a composição populacional para essa região em torno de $76 \%$. No Nordeste, o percentual de mortos foi $82 \%$ para os negros, enquanto representam $70 \%$ da população, segundo o IBGE ${ }^{40}$.

A indisponibilidade de dados segundo a raça/cor/etnia no contexto estadunidense também foi evidenciada em estudo feito de janeiro a maio de 2020 sobre vigilância de casos de Covid-19. Neste estudo, foram realizadas análises categóricas segundo sexo, faixa etária, condições de saúde e sintomas, mas não foi possível fazer comparações, análises de incidência, proporções por raça e etnia devido à elevada prevalência de dados subnotificados por essa variável ${ }^{41}$.

A divulgação de dados oficiais com baixa qualidade impossibilita o adequado monitoramento dos casos, o planejamento e a avaliação de condutas clínicas e epidemiológicas, e vigilância em saúde direcionada a contribuir para a saúde da população. Ademais, a disponibilização de dados propicia a produção de conhecimento científico e social. Logo, é fundamental a divulgação de dados com qualidade, transparência e em tempo hábil para subsidiar a construção de estratégias e a tomada de decisão no enfrentamento de uma pandemia. Os estados brasileiros que não divulgaram dados da Covid-19 por raça/cor apresentaram diferentes justificativas para não fazê-lo ${ }^{\mathbf{4 2}}$. Nesse sentido, faz-se essencial maior transparência e divulgação dos dados pelos órgãos responsáveis, de modo a assegurar o registro da variável raça/cor/etnia no processo de testagem, registros de hospitalização e de óbito. Concomitantemente, faz-se necessária a ampliação da cobertura de testagem, incluindo as comunidades quilombolas, população idosa, em situação de rua e privada de liberdade, bem como a população negra com comorbidades ${ }^{6,7}$.

No Brasil, a inclusão da variável raça/cor da pele nos sistemas de informação em saúde decorreu de intensa mobilização do movimento negro e de outros segmentos da sociedade civil na arena política. Em 2017, foi publicada a Portaria $n^{\circ} 344$, que dispõe sobre a compulsoriedade dos profissionais de saúde em coletar e preencher o campo raça/cor, respeitando-se o critério de autodeclaração ${ }^{13}$. Todavia, no caso da pandemia da Covid-19, tanto o governo brasileiro como o governo dos EUA tiveram que ser interpelados por entidades de classe, associações e movimentos negros para que os casos de Covid-19, segundo raça/cor/etnia, fossem divulgados. Coincidentemente, esses dados começaram a ser publicados nos dois países ao mesmo tempo (segunda semana do mês de abril de 2020), porém, com a mesma baixa qualidade, caracterizada por informações desencontradas, de difícil interpretação, alto índice de casos ignorados ou sem informação.

Os dados quando confiáveis, padronizados e metodologicamente bem analisados são cruciais para o aprimoramento do sistema de saúde, pois auxiliam gestores no planejamento e nas ações, apontam evidências, padrões de adoecimento e revelam se o direcionamento de recursos, as políticas e os programas estão sendo adequados e exitosos. Ademais, não existem sistemas de saúde fortes que não estejam centralizados em Sistema de Informação em Saúde (SIS) ${ }^{\mathbf{4 3}}$. No Brasil, observa-se que o gerenciamento da qualidade da informação dos SIS se dá de forma pontual, fragmentada, pouco organizada e pouco sistematizada; e, geralmente, o ciclo de produção da informação é avaliado parcialmente ${ }^{44}$.

De modo geral, as taxas de doença e morte por Covid-19 para a população negra têm sido de duas a três vezes maior do que para brancos. No tocante aos resultados parciais observados na pesquisa PNAD-Covid-19, ficou evidente maior impacto negativo da infecção pelo novo coronavírus para a população negra quando comparada à população branca. Esse fato pode decorrer da determinação social do binômio saúde-doença, da alta prevalência de doenças crônicas na população negra, das diferenças no acesso a hospitais públicos ou privados com estrutura para atender a população. Além disso, é preciso lembrar que apesar de o SUS 
ser 'universal', é muito mais utilizado pelo estrato mais carente da população brasileira, como, por exemplo, a população negra que, muitas vezes, o tem como única alternativa. No entanto, parece que isso não tem sido levado em conta pelos poderes públicos, já que o SUS vem sendo precarizado $\mathbf{4 5 , 4 6}$.

Nos EUA, a população indígena é o segmento com a maior taxa de infecção pelo novo coronavírus. No Brasil, há insuficiência de dados sobre essa população em relação à Covid-19. Todavia, pesquisa recente evidenciou que a população indígena brasileira moradora em centros urbanos teve maior proporção de pessoas infectadas pelo coronavírus $(6,3 \%)^{40}$.

O enfrentamento da pandemia da Covid-19 requer igualmente o enfrentamento das desigualdades nesses dois países. A redução das desigualdades exigirá esforços dos Estados, agências de cooperação técnica, comunidades envolvidas, participação da sociedade civil, cooperação internacional, elaboração de programas e políticas específicas. É improvável que se alcancem progressos expressivos na redução das desigualdades raciais e étnicas em saúde sem uma base científica sólida e sem iniciativas amplas, sustentáveis e longitudinais que contemplem os indicadores sociais, políticos e econômicos ${ }^{47}$. A inacessibilidade e a escassez de dados qualificados no que concerne à questão étnico-racial sobre as pessoas infectadas ou mortas pela Covid-19 representam um problema e uma grande preocupação no Brasil e nos EUA, haja vista que, para os dados publicizados até então, constata-se que grupos populacionais historicamente vulnerabilizados são os mais acometidos ${ }^{48}$.

Com vistas a viabilizar estratégias e políticas públicas verdadeiramente promotoras da equidade, enfatiza-se que, além da necessidade de qualificar os dados sobre raça/cor/etnia, há que se considerar também: faixa etária, local de moradia, tipo de residência, acesso a saneamento básico, ocupação ${ }^{18}$, entre outros determinantes sociais10; pois sabe-se que esses fatores impactam no modo de adoecer e morrer pela Covid-1948.

É importante destacar que houve retardo, por parte do Estado, na inserção da variável em tela nos instrumentos de coleta e nos boletins. Contudo, a qualidade da alimentação dos instrumentos dos sistemas de informação também depende da qualificação e da sensibilização dos profissionais de saúde que atuam nos mais variados níveis de atenção em saúde. A quantidade de registro ‘sem informação' está muito relacionada com esse aspecto.

Por fim, cabe denunciar a falta de governança para a gestão da Covid-19 no Brasil adiante da inexistência de uma coordenação nacional no Ministério da Saúde, da rotatividade da principal autoridade institucional responsável pela saúde - o Ministro da Saúde -, e da manutenção, ainda que interinamente, de uma liderança que não tem formação específica na área da saúde. Essa condição agrava e aprofunda a gestão desta crise sanitária no território brasileiro. A limitação deste estudo refere-se à dificuldade de obtenção de dados desagregados por raça/cor, qualificados, o que impossibilitou a realização de análises mais robustas.

\section{Considerações finais}

Brasil e EUA contam com população negra proporcionalmente diferente. Porém, a despeito de diferenciais numéricos, o tratamento que negros recebem nos dois países revela o racismo antinegro como um sistema excludente. Muito mais que a negação dos dados sob perspectiva étnico-racial, nega-se o reconhecimento dos sujeitos, sua identidade, seus direitos e suas necessidades.

A baixa qualidade dos dados em saúde referentes à morbimortalidade da população negra por Covid-19 reafirma o racismo e, como consequência, potencializa a vulnerabilidade desse grupo populacional. Por outro lado, o preenchimento adequado e a divulgação dos dados por raça/cor/etnia nos formulários de saúde e nos sistemas de informações em saúde do Brasil e dos EUA contribuirão para a implementação de políticas e de ações de saúde efetivas, inclusivas, bem direcionadas, com 
vistas à redução de iniquidades.

Este estudo sugere que a divulgação de dados oficiais de saúde, segundo raça/cor/ etnia, com alta proporção de dados ignorados, principalmente em um momento de pandemia, pode representar o descaso dos poderes públicos com a equidade e a prática de racismo institucional.

\section{Colaboradores}

Araujo EM (0000-0003-1643-2054)*, Caldwell KL (0000-0002-1156-7040)* e Santos MPA (0000-0003-0349-8521)* contribuíram para concepção, planejamento, análise, interpretação dos dados, elaboração do rascunho e revisão crítica do conteúdo; e participaram na aprovação da versão final do manuscrito. Souza IM (0000-0001-8481-0227)* contribuiu substancialmente para a concepção, planejamento e para a análise e a interpretação dos dados. Santa Rosa PLF (0000-0003-48320700)* contribuiu para análise e interpretação dos dados; elaboração do rascunho e revisão crítica do conteúdo; participação na aprovação da versão final do manuscrito. Santos ABS (0000-0003-3755-021X)* contribuiu para elaboração do rascunho e revisão crítica do conteúdo; e participou da aprovação da versão final do manuscrito. Batista LE (0000-00034306-3426)* contribuiu para elaboração do artigo nas seguintes atividades: revisão crítica de importante conteúdo intelectual.

\section{Referências}

1. Organização Pan-Americana da Saúde. OMS declara emergência de saúde pública de importância internacional por surto de novo coronavírus [internet]. 2020. [acesso em 2020 jul 9]. Disponível em: https://www.paho.org/bra/index.php?option=com content\&view=article $\& i d=6100: 0 \mathrm{~ms}-$ declara-emergencia-de-saude-publica-de-importancia-internacional-em-relacao-a-novo-coronavirus\&Itemid $=812$.

2. Brasil. Ministério da Saúde. Boletins Epidemiológicos (1-21) Coronavírus (COVID-19) [internet]. 2020. [acesso em 2020 jul 8]. Disponível em: https://coronavirus.saude.gov.br/boletins-epidemiologicos.
*Orcid (Open Researcher and Contributor ID).
3. Laurencin CT, McClinton A. The COVID-19 Pandemic: a Call to Action to Identify and Address Racial and Ethnic Disparities. J Racial Ethn Heal Disparities. 2020; (7):398-402.

4. Werneck J. Racismo institucional e saúde da população negra. Saúde e Soc. 2016; (25):535-49.

5. Williams DR, Priest N. Racismo e Saúde: um corpus crescente de evidência internacional. Sociologias. 2015; (17):124-74.

6. Goes EF, Ramos DO, Ferreira AJF. Desigualdades raciais em saúde e a pandemia da Covid-19. Trab Educ e Saúde. 2020; 18. 
7. Ivers LC, Walton DA. COVID-19: Global Health Equity in Pandemic Response. Am J Trop Med Hyg. 2020; (102):1149-50.

8. Araújo EM. Projeto: Análise de políticas de saúde no Brasil. [Entrevista ao Centro de Documentação e Análise Política em Saúde]. Instituto de Saúde Coletiva da Universidade Federal da Bahia. 2019 abr.; p. $1-4$.

9. Santos JR. O que é racismo? São Paulo: Brasiliense; 2005.

10. Organização Mundial da Saúde. Comissão para os Determinantes Sociais da Saúde. Redução das desigualdades no período de uma geração: igualdade na saúde através da acção sobre os seus determinantes sociais: relatório final [internet]. Genebra: OMS; 2010. [acesso em 2020 jul 8]. Disponível em: https://www. who.int/eportuguese/publications/Reducao_desigualdades_relatorio2010.pdf.

11. Alves JAL. A Conferência de Durban contra o Racismo e a responsabilidade de todos. Rev Bras Política Int. 2002; (45):198-223.

12. Brasil. Ministério da Saúde. Política Nacional de Saúde Integral da População Negra. 2. ed. Brasília, DF: MS; 2013.

13. Brasil. Ministério da Saúde. Portaria no 344 , de $1^{\circ}$ de fevereiro de 2017. Dispõe sobre o preenchimento do quesito raça/cor nos formulários dos sistemas de informação em saúde. Brasília; 2017. Diário Oficial da União. 1 Fev 2017.

14. Brasil. Ministério da Saúde. Portaria no 992, de 13 de maio de 2009. Institui a Política Nacional de Saúde Integral da População Negra. 2009. Diário Oficial da União. 13 Maio 2009.

15. Souza IM, Hughes GD, van Wyk BE, et al. Comparative Analysis of the Constitution and Implementation of Race/Skin Color Field in Health Information Systems: Brazil and South Africa. J Racial Ethn Heal Disparities. 2020; 1-13.
16. Boyle K. Dimensions of Racism. In: Proceedings of a Workshop to commemorate the end of the United Nations Third Decade to Combat Racism and Racial Discrimination. New York; Geneva: United Nations; 2005. p. 1-12.

17. Center for Disease Control and Prevention. Use of Race and Ethnicity in Public Health Surveillance. Summary of the CDC/ATSDR Workshop. MMWR. 1993; 42(RR-10):1-16.

18. Barber S, Diez Roux AV, Cardoso L, et al. At the intersection of place, race, and health in Brazil: Residential segregation and cardio-metabolic risk factors in the Brazilian Longitudinal Study of Adult Health (ELSA-Brasil). Soc Sci Med. 2018; (199):67-76.

19. Instituto Brasileiro de Geografia e Estatística. Pesquisa Nacional por Amostra de Domicílios PNAD/ COVID19. PNAD [internet]. 2020. [acesso em 2020 jul 7]. Disponível em: https://www.ibge.gov.br/estatisticas/sociais/saude/27946-divulgacao-semanal-pnadcovidl.html?=\&t=resultados.

20. Instituto Brasileiro de Geografia e Estatística. Projeção da população do Brasil e das Unidades da Federação. População brasileira às 16:13:51 de 21/4/2020 [internet]. 2020. [acesso em 2020 jun 18]. Disponível em: https:// www.ibge.gov.br/apps/populacao/projecao/.

21. Osório RG. O sistema classificatório de "cor ou raça" do IBGE. Brasília, DF: IBGE; 2003.

22. Programa das Nações Unidas para o Desenvolvimento. Relatório do Desenvolvimento Humano 2019. Além do rendimento, além das médias, além do presente: Desigualdades no desenvolvimento humano no século XXI. New York: PNUD; 2019.

23. Brasil. Constituição, 1988. Constituição da República Federativa do Brasil. Brasília, DF: Senado Federal; 1988.

24. United States Census Bureau: Tabela de dados sobre a população (2010-2019) [internet]. 2020 [acesso em 2020 jul 8]. Disponível em: https://www.census.gov/ quickfacts/fact/table/US/PST045219. 
25. United States Government. Public Law 111-148. 111th United States Congress [internet]. Washington, DC: USG; 2010. [acesso em 2020 jul 10]. Disponível em: https://www.congress.gov/111/plaws/publ148/PLAW-111publ148.pdf.

26. CNN. Obamacare: Enrollment numbers and Medicaid expansion [internet]. CNN. 2014 maio 5. [acesso em 2014 jun 21]. Disponível em: http://www.cnn. com/interactive/2013/09/health/map-obamacare/.

27. Artiga S, Orgera K, Damico A. Changes in Health Coverage by Race and Ethnicity since the ACA, 20102018 - Issue Brief. Kaiser Family Foundation [internet]. 2020. [acesso em 2020 jul 6]. Disponível em: https://www.kff.org/disparities-policy/issue-brief/ changes-in-health-coverage-by-race-and-ethnicity-since-the-aca-2010-2018/.

28. Gaba C, Gee E. How Trump's Policies Have Hurt ACA Marketplace Enrollment. Center for American Progress [internet]. 2020. [acesso em 2020 jul 6]. Disponível em: https://www.americanprogress.org/issues/ healthcare/news/2020/04/16/483362/trumps-policies-hurt-aca-marketplace-enrollment/.

29. Inequality.org. Racial Inequality and Covid-19 [internet]. [acesso em 2020 jul 8]. Disponível em: https:// inequality.org/facts/racial-inequality/.

30. Center for Disease Control and Prevention. COVID-19 in Racial and Ethnic Minority Groups [internet]. 2020. [acesso em 2020 jul 9]. Disponível em: https://www. cdc.gov/coronavirus/2019-ncov/need-extra-precautions/racial-ethnic-minorities.html.

31. Lardieri A, Writer S. Hispanics and Blacks Are Hardest Hit by COVID-19 in New York City. U.S. News \& World Report [internet]. 2020. [acesso em 2020 jul 9]. Disponível em: https://www.usnews.com/news/ national-news/articles/2020-04-08/coronavirus-disproportionally-kills-hispanics-and-blacks-in-new-york-city.

32. New York. New York State Department of Health. COVID-Fatalities [internet]. 2020. [acesso em 2020 jun 30]. Disponível em: https://covid19tracker.heal-
th.ny.gov/views/NYS-COVID19-Tracker/NYSDOHCOVID-19Tracker-Fatalities?\%3Aembed=yes\&\% 3Atoolbar $=$ no\&\%3Atabs $=$ n.

33. Millett GA, Jones AT, Benkeser D, et al. Assessing differential impacts of COVID-19 on black communities. Ann Epidemiol. 2020; (47):37-44.

34. Gordon C, Johnson W, Purnell JQ, et al. COVID-19 and the Color Line [internet]. Boston Review. A Political and Literary Forum. 2020 Maio 1. [acesso em 2020 jul 6]. Disponível em: http://bostonreview.net/ race/colin-gordon-walter-johnson-jason-q-purnell-jamala-rogers-covid-19-and-color-line.

35. Center for Disease Control and Prevention. Age Adjusted COVID-19-associated hospitalization rates by race and ethnicity, COVID-NET, March 1-May 23 [internet]. 2020 [acesso em 2020 jun 30]. Disponível em: https://www.cdc.gov/coronavirus/2019-ncov/covid-data/images/us-mortality-graph-animated-06032020.jpg.

36. Johns Hopkins. Johns Hopkins University \& Medicine. Racial Data Transparency States that have released breakdowns of Covid-19 data by race [internet]. 2020. [acesso em 2020 jul 8]. Disponível em: https:// coronavirus.jhu.edu/data/racial-data-transparency.

37. Guimarães ASA. Combatendo o racismo: Brasil, África do Sul e Estados Unidos. Rev Bras Ciências Sociais. 1999; (14):103-15.

38. Nogueira O. Tanto preto quanto branco: estudos de relações raciais. São Paulo: T.A. Queiroz; 1985.

39. Instituto Brasileiro de Geografia e Estatística. População chega a 205,5 milhões, com menos brancos e mais pardos e pretos [internet]. Agência IBGE Notícias. 2019 fev 12. [acesso em 2020 mar 11]. Disponível em: https://agenciadenoticias.ibge.gov.br/agencia-noticias/2012-agencia-de-noticias/noticias/18282-populacao-chega-a-205-5-milhoes-com-menos-brancos-e-mais-pardos-e-pretos.

40. Soares M. Dados do SUS revelam vítima-padrão da COVID-19 no Brasil: homem, pobre e negro[internet]. 
Revista Época. 2020 jul 13. [acesso em 2020 jun 8]. Disponível em: https://epoca.globo.com/sociedade/ dados-do-sus-revelam-vitima-padrao-de-covid-19-no-brasil-homem-pobre-negro-24513414.

41. Stokes EK, Zambrano LD, Anderson KN, et al. Coronavirus Disease 2019 Case Surveillance - United States, January 22-May 30, 2020. MMWR Morb Mortal Wkly Rep. 2020; (69):759-65.

42. Faria F, Yukari D, Velasco C, et al. Maioria dos estados não divulga dados sobre cor de vítimas da Covid-19 [internet]. Folha São Paulo. 2020 jun 21. [acesso em 2020 jul 8]. Disponível em: https://wwwl.folha.uol. com.br/equilibrioesaude/2020/06/maioria-dos-estados-nao-divulga-dados-sobre-raca-de-vitimas-da-covid-19.shtml.

43. Moucheraud C, Schwitters A, Boudreaux C, et al. Sustainability of health information systems: a three-country qualitative study in southern Africa. BMC Health Serv Res. 2017; (17):23.

44. Correia LOS, Padilha BM, Vasconcelos SML. Métodos para avaliar a completitude dos dados dos siste- mas de informação em saúde do Brasil: uma revisão sistemática. Ciênc. Saúde Colet. 2014; (19):4467-78.

45. Paixão M, Rossetto I, Montovanele F, et al. Relatório anual das desigualdades raciais no Brasil 2009-2010 - Constituição Cidadã, seguridade social e seus efeitos sobre as assimetrias de cor ou raça. Rio de Janeiro: Garamond; 2011.

46. Giovanella L, Mendoza-Ruiz A, Pilar ACA, et al. Sistema universal de saúde e cobertura universal: desvendando pressupostos e estratégias. Ciênc. Saúde Colet.2018; (23):1763-76.

47. Williams DR, Lawrence JA, Davis BA. Racism and Health: Evidence and Needed Research. Annu Rev Public Health. 2019; (40):105-25.

48. Santos MPA, Nery JS, Goes EF, et al. População negra e Covid-19: reflexões sobre racismo e saúde. Estud Avançados. 2020; (34):225-43.

Recebido em 14/07/2020

Aprovado em 19/09/2020

Conflito de interesses: inexistente

Suporte financeiro: não houve 